\title{
Oral Aspects of Metabolic Disorders
}

\section{Ankita Bohra* and Bhateja S}

P.G Oral Medicine and Radiology Department, Vyas Dental College and Hospital, Jodhpur, India

*Corresponding author:Ankita Bohra, P.G Oral Medicine and Radiology Department, Vyas Dental College and Hospital, Jodhpur, India, Tel: + 919414454413; E-mail: aav1423@hotmail.com

Rec Date: June 25, 2015; Acc Date: August 13, 2015; Pub Date: August 17, 2015

Copyright: (C) 2015 Bohra A et al. This is an open-access article distributed under the terms of the Creative Commons Attribution License, which permits unrestricted use, distribution, and reproduction in any medium, provided the original author and source are credited.

\section{Abstract}

Metabolism is a complex process that involves a series of chemical reactions in the human body. Alterations in these metabolic processes constitute the disturbances of metabolism causing metabolic disorders. The breakdown of carbohydrate by the oral microorganisms has been the subject of numerous investigations. This review discusses the oral aspects of metabolic diseases.

Keywords: Metabolism; Oral flora; Metabolic disorder

\section{Introduction}

\section{Metabolism}

Duncan defined metabolism as "the sum total of tissue activity as considered in terms of physicochemical changes associated with and regulated by the availability, utilization and disposal of protein, fat, carbohydrate, vitamins, minerals, water and the influences which the endocrines exert on these processes". Metabolism is a complex process that involves a series of chemical reactions in the human body. Some reactions produce the energy which is stored in the form of ATP while other reactions consume energy to manufacture complex compounds. The process of metabolism includes 2 types of mechanisms, i.e, catabolism and anabolism [1].

Catabolism is a process which involves release of energy by breaking complex organic compounds into simple molecules. Thus, in this process energy is been produced.

Anabolism is a process that requires energy for synthesis of complex compounds from small molecules. In this process energy is been utilized.

Alterations in these metabolic processes constitute the disturbances of metabolism causing metabolic disorders. The breakdown of carbohydrate by the oral microorganisms has been the subject of numerous investigations [2]. It is believed by many that the intraoral production of acids from carbohydrate, arising from incomplete oxidation, is a factor in the production of dental caries, periodontal diseases, oral malodor, bone loss and other associated metabolic diseases [3].

\section{Types of Metabolic Disorders [4,5]}

- Disturbances in Protein metabolism

- Disturbances in Lipid metabolism

- Disturbances in Carbohydrate metabolism

- Disturbances in hormone metabolism

- Lysosomal storage disorders

\section{- Mitochondrial storage disorders}

\section{Disturbances of protein metabolism}

Amyloidosis: It is a rare disease that results from accumulation of inappropriately folded proteins. These misfolded proteins are called amyloids. When proteins that are normally soluble in water fold to become amyloids, they become insoluble and deposit in organs or tissues, disrupting normal function.

Protein energy malnutrition: PEM includes Kwashiorkor (protein malnutrition predominant), Marasmus (deficiency in calorie intake), Marasmic Kwashiorkor (marked protein deficiency and marked calorie insufficiency signs present, sometimes referred to as the most severe form of malnutrition) PEM is fairly common worldwide in both children and adults and accounts for 6 million deaths annually. PEM may be secondary to other conditions such as chronic renal disease or cancer cachexia in which protein energy wasting may occur.

Porphyria: The porphyrias are a group of rare diseases in which chemical substances called porphyrins accumulate. The body requires porphyrins to produce heme, which carries oxygen in the blood; but, in the porphyrias, there is a deficiency (inherited or acquired) of the enzymes that transform the various porphyrins into others, leading to abnormally high levels of one or more of these substances. This manifests with either neurological complications or skin problems or occasionally both.

Erythropoietic uroporphyria: It is caused by genetic defects which lead to deficiency of the enzyme uroporphyrinogen III cosynthase (UROS). The disease is characterised by extreme photosensitivity (abnormal cutaneous reaction to sunlight) which can leave severe scarring, blister formation and the loss of digits or other features. Damaged skin can become infected, leading to further necrosis and deformities. The face, hands and arms are the most significantly affected as they are frequently exposed; sometimes presenting as severe disfiguration.

\section{Disturbances in carbohydrate metabolism [6]}

Mucopolysacchridoses result from abnormal degradation of glycosaminoglycans. Disorders in carbohydrate metabolism include Mucopolysacchridoses (MPS) type I, II, III, IV, VIa, VIb, VII. 
MPS Type I includes Hurler and Scheie syndrome: It is a cutaneous condition characterized by mild mental retardation and corneal clouding (Figure 1).

MPS Type II includes Hunter syndrome: (mild to severe form) It is a lysosomal storage disease caused by a deficient or absent enzyme, iduronate-2-sulfatase (I2S). The accumulated substrates in Hunter syndrome are heparan sulfate and dermatan sulfate. The syndrome has $\mathrm{X}$-linked recessive inheritance.

MPS Type III includes Sanfillippo syndrome: It is a metabolism disorder passed down through families. It makes the body unable to properly break down long chains of sugar molecules called glycosaminoglycans (formerly called mucopolysaccharides). The syndrome belongs to a group of diseases called mucopolysaccharidoses (MPS).

MPS Type IV includes Morquio syndrome: It is an autosomal recessive mucopolysaccharide storage disease usually inherited. It is a rare type of birth defect with serious consequences like cardiovascular and ophthalmic defects.

MPS Type VIa includes Maroteaux- Lamy syndrome (classic form) MPS Type VIb includes Maroteaux- Lamy syndrome (mild form): It is a form of mucopolysaccharidosis caused by a deficiency in arylsulfatase B (ARSB). Symptoms include neurological complications like clouded corneas, deafness, thickening of the dura and pain caused by compressed or traumatized nerves and nerve roots.

MPS Type VII includes beta glucuronidase deficiency: It is a very rare genetic disorder with high mortality rate. Congenital defect in multiple organs with gender predilection for males.

\section{Mucopolysaccharidosis I (MPS I) Disease (Hurler, Hurler-Scheie, Scheie Syndromes)}

\section{SYMPTOMES IMAGES OF KEY FEATURES}

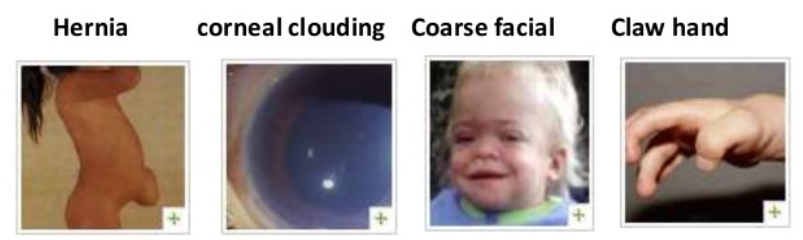

Figure 1: Showing key features of Hurler syndrome.

\section{Disturbances in lipid metabolism [7]}

Gaucher's disease: It is a genetic disease in which glucosylceramide accumulate in cells and certain organs. The disorder is characterized by bruising, fatigue, anaemia, low blood platelets, and enlargement of the liver and spleen. It is caused by a hereditary deficiency of the enzyme glucocerebrosidase.

Niemann-Pick disease: It is one of a group of lysosomal storage diseases that affect metabolism and that are caused by genetic mutations. Enlargement of the liver and spleen (hepatosplenomegaly) may cause reduced appetite, abdominal distension, and pain.

Letterer-Siwe disease: It is the acute disseminated multisystem form of Langerhans cell histiocytosis characterised by proliferation of nonlipid histiocytes in the viscera and bones. Clinical features include a variety of skin lesions, osteolytic lesions, lymphadenopathy, hepatosplenomegaly, pulmonary infiltration, spiking fever, anaemia, thrombocytopenia, mandibular hyperplasia, gingival inflammation, loss of teeth, otitis media, hemorrhages, failure to thrive, cachexia.

\section{Disturbances in Hormone metabolism [8]}

Pituitary hormone: Hypopituitarism: Symptoms include fatigue, low blood pressure, weight loss, weakness, depression, nausea, or vomiting, constipation, weight gain, sensitivity to cold, decreased energy, and muscle weakness or aching. In women, symptoms include irregular or stopped menstrual periods and infertility. Hyperpituitarism: Symptoms includes headaches, visual disturbance, and growth failure, weight gain. Hirsutism and premature adrenarche may occur in prepubertal children. Pubertal arrest, acne, fatigue, and depression are also common.

Thyroid hormone: Hyperthyroidim includes irritability, muscle weakness, sleeping problems, a fast heartbeat, poor tolerance of heat, diarrhea, enlargement of the thyroid gland, exopthalamus and weight loss. Hypothyroidsm includes poor ability to tolerate cold, a feeling of tiredness, and weight gain. In children, hypothyroidism leads to delays in growth and intellectual development which is called cretinism.

Parathyroid hormone: Hyperparathyroidism includes depression, fatigue, polydypsia and polyuria, feeling sick and losing your appetite, muscle weakness, constipation, tummy pain, loss of concentration. Hypoparathyroidism includes muscle aches or cramps, tingling, burning, or numbness in fingertips, toes, and lips, muscle spasms, especially around the mouth, patchy hair loss, dry skin, brittle nails, fatigue, anxiety or depression.

\section{Lysosomal storage disorders $[9,10]$}

Lysomal storage disorders are a group of approximately 50 rare inherited metabolic disorders results from defects in lysosomal function. Lysosomes are sacs of enzymes within cells that digest large molecules and pass the fragments on to other parts of the cell for recycling. This process requires several critical enzymes. If one of these enzymes is defective, because of a mutation, the large molecules accumulate within the cell, eventually killing it. The symptoms of lysosomal storage disease vary, depending on the particular disorder and other variables like the age of onset, and can be mild to severe. They include developmental delay, movement disorders, seizures dementia, deafness and/or blindness, hepatomegaly splenomegaly and cardiac problems, and bones that grow abnormally. Some examples are listed below.

Schinder disease/Kanzaki disease: Schindler disease results from the deficient activity of the enzyme alpha- $N$-acetylgalactosaminidase (alpha-galactosidase B), with the accumulation of sialylated-asialoglycopeptide and oligosaccharide with alpha- $N$-acetylgalactosamilnyl residues.

Faber disease: (Farber's lipogranulomatosis, ceramidase deficiency) is an extremely rare ( 80 cases reported worldwide to this day) autosomal recessive lysosomal storage disease marked by a deficiency in the enzyme ceramidase that causes an accumulation of 
Page 3 of 4

fatty material lipids leading to abnormalities in the joints, liver, throat tissues and central nervous system.

Krabbe disease: (globoid cell leukodystrophy) is a rare, often fatal degenerative disorder that affects the myelin sheath of the nervous system. It is a form of sphingolipidosis, as it involves dysfunctional metabolism of sphingolipids. This condition is inherited in an autosomal recessive pattern.

Tay-Sachs and Sandhoff diseases: is a rare autosomal recessive genetic disorder. It causes a progressive deterioration of nerve cells and of mental and physical abilities that begins around six months of age and usually results in death by the age of four.

Pyknodysostosis: also known as osteopetrosis acroosteolytica or Toulouse-Lautrec syndrome is a rare autosomal recessive bone dysplasia, characterised by osteosclerosis and short stature.

\section{Mitochondrial diseases [11]}

Mitochondrial disease is a group of disorders caused by dysfunctional mitochondria, the organelles that generate energy for the cell. Mitochondrial disorders may be caused by mutations, acquired or inherited, in mitochondrial DNA (mtDNA) or in nuclear genes that code for mitochondrial components. They may also be the result of acquired mitochondrial dysfunction due to adverse effects of drugs, infections, or other environmental causes. Symptoms include poor growth, loss of muscle coordination, muscle weakness, visual problems, hearing problems, learning disabilities, heart disease, liver disease, kidney disease, gastrointestinal disorders, respiratory disorders, neurological problems, autonomic dysfunction and dementia. Examples of mitochondrial diseases include-

Luft disease: Consist of Hypermetabolism, with fever, heat intolerance, profuse perspiration, polyphagia, polydipsia, ragged-red fibers, and resting tachycardia. Exercise intolerance with mild weakness.

Leigh syndrome: Consist of subacute sclerosing encephalopathy after normal development the disease usually begins late in the first year of life, although onset may occur in adulthood. A rapid decline in function occurs and is marked by seizures, altered states of consciousness, dementia, ventilatory failure (Figure 2).

Alpers disease: Also called as Progressive Infantile Poliodystrophy. Symptoms include seizures, dementia, spasticity, blindness, liver dysfunction, and cerebral degeneration.

Pearson marrow syndrome: It is a mitochondrial disease characterized by sideroblastic anaemia and exocrine pancreas dysfunction. Clinical features includes failure to thrive, pancreatic fibrosis, muscle and neurologic impairment, and, frequently, early death.

Wilson disease: is an autosomal recessive disorder in which copper accumulates in tissues this manifests as neurological or psychiatric symptoms and liver disease. It is treated with medication that reduces copper absorption or removes the excess copper from the body, but occasionally a liver transplant is required.

Batten disease: It is a rare, fatal autosomal recessive neurodegenerative disorder that begins in childhood. Early signs may be subtle personality and behaviour changes, slow learning or regression, repetitive speech or echolalia, clumsiness, or stumbling. There may be slowing head growth in the infantile form, poor circulation in lower extremities (legs and feet), decreased body fat and muscle mass, curvature of the spine, hyperventilation and/or breathholding spells, teeth grinding, and constipation.

Menkes disease: It is a disorder that affects copper levels in the body leading to copper deficiency. It is an $\mathrm{x}$ linked recessive disorder, and is therefore considerably more common in males: females require two defective alleles to develop the disease. Signs and symptoms of this disorder include weak muscle tone (hypotonia), sagging facial features, seizures, intellectual disability, blue sclera and developmental delay. The patients have brittle hair and metaphyseal widening. In rare cases, symptoms begin later in childhood and are less severe. Affected infants may be born prematurely.

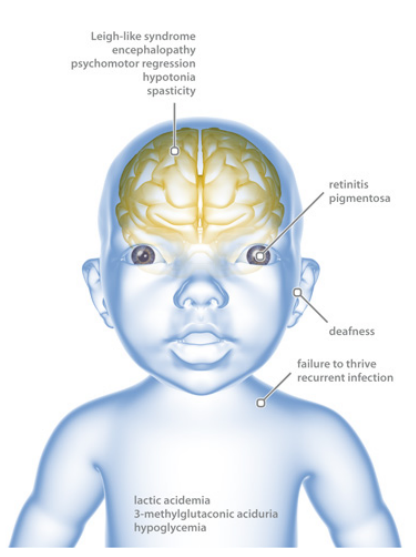

Figure 2: Showing features of Leigh syndrome.

\section{Conclusion}

Process of metabolism is very complex and intermingled with multiple factors responsible for homeostasis. Oral microbial flora also plays a vital role in this process. Any kind of change in the pathway from cellular to macroscopic level may give rise to number of metabolic diseases associated with local and systemic changes. This review enlightens various types of metabolic disorders and their associated oral conditions.

\section{References}

1. Ebenhöh O, Heinrich R (2001) Evolutionary optimization of metabolic pathways. Theoretical reconstruction of the stoichiometry of ATP and NADH producing systems. Bull Math Biol 63: 21-55.

2. Takahashi N, Sato T (2002) Dipeptide utilization by the periodontal pathogens Porphyromonas gingivalis, Prevotella intermedia, Prevotella nigrescens and Fusobacterium nucleatum. Oral Microbiol Immunol 17: 50-54.

3. Niederman R, Zhang J, Kashket S (1997) Short-chain carboxylic-acidstimulated, PMN-mediated gingival inflammation. Crit Rev Oral Biol Med 8: 269-290.

4. Takahashi N, Saito K, Schachtele CF, Yamada T (1997) Acid tolerance of growth and neutralizing activity of Porphyromonas gingivalis, Prevotella intermedia and Fusobacterium nucleatum. Oral Microbiol Immunol 12: 323-328.

5. Mayanagi G, SatoT, Shimauchi H, Takahashi N (2004) Detection frequency of periodontitis-associated bacteria by polymerase chain reaction in subgingival and supragingival plaque of subjects with periodontitis and healthy subjects. Oral Microbiol Immunol 19: 379-385. 
Page 4 of 4

6. Saito K, Takahashi N, Horiuchi H, Yamada T (2001) Effects of glucose on formation of cytotoxic end-products and proteolytic activity of Prevotella intermedia and Prevotella nigrescens. J Periodontal Res 36: 355-360.

7. Ratcliff PA, Johnson PW (1999) The relationship between oral malodor, gingivitis, and periodontitis. A review. J Periodontol 70: 485-489.

8. Winchester B, Vellodi A, Young E (2000) The molecular basis of lysosomal storage diseases and their treatment. Biochem Soc Trans 28: 150-154.
9. Ponder KP, Haskins ME (2007) Gene therapy for mucopolysaccharidosis. Expert Opin Biol Ther 7: 1333-1345.

10. Finsterer J (2007) Hematological manifestations of primary mitochondrial disorders Acta Haematol 118: 88-98.

11. Marriage B, Clandinin MT, Glerum DM (2003) Nutritional cofactor treatment in mitochondrial disorders. J Am Diet Assoc 103: 1029-1038. 